\title{
Real-time Characterization of the Optical Properties of an ultra-thin Surface Reaction Layer during Growth
}

\author{
N. Dietz ${ }^{a, b}$, N. Sukidi ${ }^{b}$, C. Harris ${ }^{b}$ and K.J. Bachmann ${ }^{a, c}$ \\ Departments of Physics ${ }^{\mathrm{a}}$, Materials Science and Engineering $\mathrm{b}$ and Chemical Engineering ${ }^{\mathrm{c}}$, North \\ Carolina State University, Raleigh, North Carolina 27696-7919
}

\begin{abstract}
The average optical properties of an ultra-thin surface reaction layer (SRL) during growth by pulsed chemical beam epitaxy (PCBE) can be quantitatively accessed by p-polarized reflectance spectroscopy (PRS), as demonstrated on the example of heteroepitaxial GaP growth. Under PCBE growth conditions, the surface of the substrate is exposed to pulsed ballistic beams of tertiarybutyl phosphine [TBP, $\left(\mathrm{C}_{4} \mathrm{H}_{9}\right) \mathrm{PH}_{2}$ ] and triethylgallium [TEG, $\mathrm{Ga}\left(\mathrm{C}_{2} \mathrm{H}_{5}\right)_{3}$ ]. The pulsed precursor supply causes a period in composition and thickness modulated SRL, monitored as a fine structure that is superimposed on interference oscillations, resulting from back reflection at the substrate-layer interface with increasing layer thickness. The amplitude of this fine structure undergoes a period amplitude modulation and exhibits turning points at which the response to the first precursor pulse changes sign. The turning points can be characterized by the expression $\mathrm{R}_{4}\left(\Phi_{\max }\right)=\mathrm{R}_{4}\left(\Phi_{\min }\right)$, which describes the maximal and minimal values of the temporally modulated phase factor in the SRL, using a four layer stack description. The positions of these turning points are not affected by the thickness of the SRL, which allows the computation of the average complex dielectric function of the SRL independent of its thickness. In the next step, the average thickness of the SRL can be extracted from the amplitude of the observed fine structure.
\end{abstract}

\section{Introduction}

For the understanding of deposition processes and the involved surface chemistry, non-intrusive optical real-time process techniques have been successfully applied during the last decade, focusing on the monitoring of either bulk-film properties[1-4] or surface processes by reflection high energy electron diffraction (RHEED), or reflectance difference spectroscopy (RDS[5]). For the characterization of both, bulk and surface, we added recently p-polarized reflectance spectroscopy (PRS) and demonstrated its capability during pulsed chemical beam epitaxy (PCBE) of III-V heteroepitaxial growth. Single wavelength PR and laser light scattering (LLS) data are obtained simultaneously during heteroepitaxial film growth under pulsed chemical beam epitaxy conditions, as described previously [6-13].

Figure 1 shows a typical evolution of the PR and LLS signals during GaP growth on a $\mathrm{Si}(001)$ substrate. The signals can be analyzed in terms of the bulk and surface properties of the film as described previously[12]. The fine structure observed in the PR signal can be understood as the optical response in a four-layer stack description, where one oscillation in the fine structure corresponds to a complete precursor cycle. The start of an oscillation coincides with the leading edge of the first precursor pulse in the cycle sequence (see insert in fig. 1). As shown in fig. 1, the amplitude in the fine structure undergoes periodic changes during deposition time. The amplitude increases on the raising flank of the interference oscillation with a maximum at the top, and then decreases on the falling flank. The relative locations of these decreases and increases in the fine structure amplitude and the film interference oscillation strongly depend on the chosen growth conditions, such as precursor pulse width and height, pulse sequence time, or supply of additional activated hydrogen. Characteristic features can be identified using the first derivative of the PR signal, shown in fig. 1 . The envelope function of $\mathrm{d}(\mathrm{PR}) / \mathrm{dt}$ reveals two important features: 
1. The minima extrema in $\mathrm{d}(\mathrm{PR}) / \mathrm{dt}$ mark the position where the response to the first precursor pulse changes sign (turning points).

2. During steady-state growth conditions, the $\mathrm{d}(\mathrm{PR}) / \mathrm{dt}$ evolution undergoes period oscillations, where one oscillation consists of a long period with a large amplitude and a short period with a small amplitude. The ratio of these periods and their amplitude strongly depends on the chosen precursor dose and exposure times.

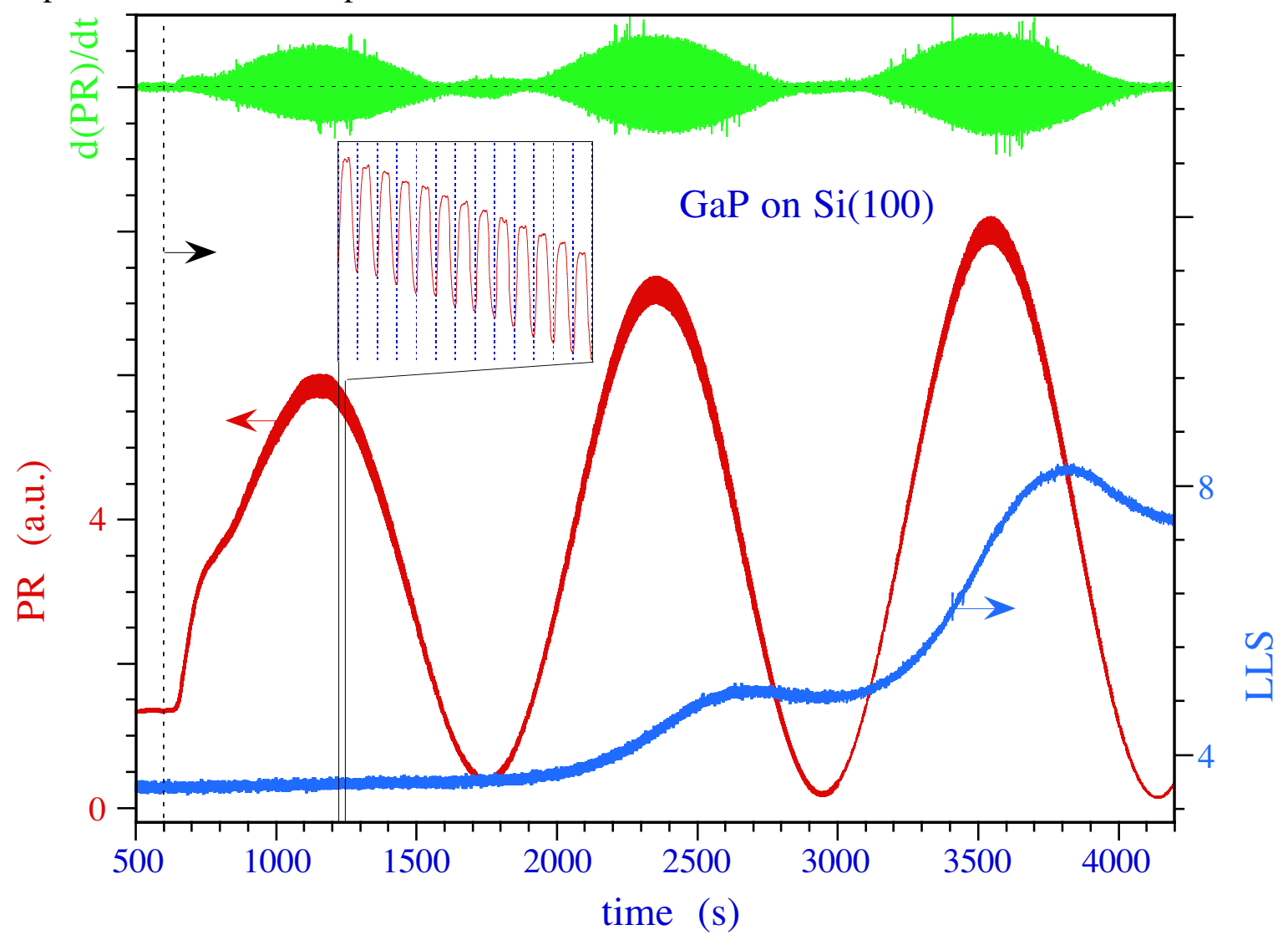

Figure 1: PR, LLS and d(PR)/dt evolution for heteroepitaxial GaP growth on Si under PCBE conditions at $350^{\circ} \mathrm{C}$. PR is monitored under an angle of $75 \mathrm{deg}$. The insert shows an enlargement of the fine structure as a response to the sequential precursor exposure of the surface.

\section{FINE STRUCTURE MODELING}

The mathematical modeling of the fine structure can be described under the simplified assumption of a periodically in phase modulated SRL with an average dielectric function $\&_{1}$ $[11,14]$. Thereby, the changes in the phase factor of the SRL are linked to the buildup of SRL with the thickness $\mathbb{d}_{1}$ by the first precursor pulse and the incorporation of the SRL in the underlying growing film after the second precursor pulse. Under these assumptions the overall observed evolution of the PR signal is described, using Fresnel's equations and a four-layer (ambient / surface_layer / film / substrate) stack model. A more detailed model has to account for changes in both thickness and dielectric function of the SRL as the growth process progresses. This requires a correlation of structure in the PR intensity to changing of defragmentation products and their concentrations in the surface reaction layer, which is discussed in more detail elsewhere [15].

Fresnel's equations for a multilayer stack are used to calculate the changes of the reflectivity for p-polarized light as a function of layer thickness, assuming homogenous isotropic media. 
Numbering the media forming the multilayer stack through, the reflectance coefficients $r_{k(k+1)}$ for the interfaces are given by

$$
\mathrm{r}_{\mathrm{k}(\mathrm{k}+1)}=\frac{\varepsilon_{\mathrm{k}+1} \bullet \sqrt{\varepsilon_{\mathrm{k}}-\varepsilon_{0} \sin ^{2} \varphi}-\varepsilon_{\mathrm{k}} \cdot \sqrt{\varepsilon_{\mathrm{k}+1}-\varepsilon_{0} \sin ^{2} \varphi}}{\varepsilon_{\mathrm{k}+1} \bullet \sqrt{\varepsilon_{\mathrm{k}}-\varepsilon_{0} \sin ^{2} \varphi}+\varepsilon_{\mathrm{k}} \bullet \sqrt{\varepsilon_{\mathrm{k}+1}-\varepsilon_{0} \sin ^{2} \varphi}} \quad(\mathrm{k} \geq 0)
$$

Here, in the four layer stack model used, ' 0 ' denotes the ambient, ' 1 ' the surface reaction layer, ' 2 ' the film, and ' 3 ' the substrate, with their complex dielectric constants $\varepsilon_{0}, \varepsilon_{1}, \varepsilon_{2}$ and $\varepsilon_{3}$, respectively. The complex reflectance amplitude $\mathrm{rr}_{4}$ for $\mathrm{p}$-polarized light is given by

$$
\mathrm{rr}_{4}=\frac{\mathrm{r}_{01}+\mathrm{r}_{12} \mathrm{e}^{-2 \mathrm{j} \Phi_{1}}+\mathrm{r}_{23} \mathrm{e}^{-2 \mathrm{j}\left(\Phi_{1}+\Phi_{2}\right)}+\mathrm{r}_{01} \mathrm{r}_{12} \mathrm{r}_{23} \mathrm{e}^{-2 \mathrm{j} \Phi_{2}}}{1+\mathrm{r}_{01} \mathrm{r}_{12} \mathrm{e}^{-2 \mathrm{j} \Phi_{1}}+\mathrm{r}_{01} \mathrm{r}_{23} \mathrm{e}^{-2 \mathrm{j}\left(\Phi_{1}+\Phi_{2}\right)}+\mathrm{r}_{12} \mathrm{r}_{23} \mathrm{e}^{-2 \mathrm{j} \Phi_{2}}}
$$

with the phase factors

$$
\Phi_{1}(\mathrm{t})=\frac{2 \pi \mathrm{d}_{1}(\mathrm{t})}{\lambda} \sqrt{\varepsilon_{1}(\mathrm{t})-\varepsilon_{0} \sin ^{2} \varphi}
$$

and

$$
\Phi_{2}(\mathrm{t})=\frac{2 \pi \mathrm{d}_{2}(\mathrm{t})}{\lambda} \sqrt{\varepsilon_{2}-\varepsilon_{0} \sin ^{2} \varphi} .
$$

$d_{1}(t)$ and $d_{2}(t)$ are the thicknesses of surface reaction layer (SRL) and film, respectively, $\lambda$ is the wavelength of the reflected p-polarized laser beam and $\varphi$ the angle of incidence. Since only $\mathrm{p}$ polarized light is considered in the following, the index $\mathrm{p}$ is dropped for simplification.

In the analysis of the temporal evolution of the fine structure that is superimposed on the interference oscillations of the underlying growing film, the different time evolution of the both phase factors $\Phi_{1}$ and $\Phi_{2}$ can be utilized to separate contributions related to the SRL and thus related to the bulk film. Assuming time dependencies in $d_{2}(\boldsymbol{I}), d_{1}(t)$ and $\varepsilon_{1}(t)$, the derivative of $R_{4}$ in time is given by

$$
\begin{aligned}
\frac{\mathrm{d} \mathrm{R}_{4}}{\mathrm{dt}}= & \frac{\partial \mathrm{R}_{4}}{\partial \phi_{2}} \bullet \frac{\partial\left(\phi_{2}\right)}{\partial \mathrm{t}}+\frac{\partial \mathrm{R}_{4}}{\partial \phi_{1}} \bullet \frac{\partial\left(\phi_{1}\right)}{\partial \mathrm{t}}+\left[\frac{\partial \mathrm{R}_{4}}{\partial \mathrm{r}_{12}} \bullet \frac{\partial \mathrm{r}_{12}}{\partial \varepsilon_{1}}+\frac{\partial \mathrm{R}_{4}}{\partial \mathrm{r}_{01}} \bullet \frac{\partial \mathrm{r}_{01}}{\partial \varepsilon_{1}}\right] \bullet \frac{\partial\left(\varepsilon_{1}\right)}{\partial \mathrm{t}} \\
\approx \frac{\partial \mathrm{R}_{3}(\boldsymbol{f})}{\partial \mathfrak{f}} & +\left[\frac{\partial\left[\Delta \mathrm{R}_{4-3}\right]}{\partial \phi_{1}} \bullet \frac{\partial \phi_{1}}{\partial \mathrm{d}_{1}}\right] \bullet \frac{\partial\left[\mathrm{d}_{1}(\mathrm{t})\right]}{\partial \mathrm{t}} \\
& +\left[\frac{\partial\left[\Delta \mathrm{R}_{4-3}\right]}{\partial \mathrm{r}_{12}} \bullet \frac{\partial \mathrm{r}_{12}}{\partial \varepsilon_{1}}+\frac{\partial\left[\Delta \mathrm{R}_{4-3}\right]}{\partial \mathrm{r}_{01}} \bullet \frac{\partial \mathrm{r}_{01}}{\partial \varepsilon_{1}}+\frac{\partial\left[\Delta \mathrm{R}_{4-3}\right]}{\partial \phi_{1}} \bullet \frac{\partial \phi_{1}}{\partial \varepsilon_{1}}\right] \bullet \frac{\partial\left[\varepsilon_{1}(\mathrm{t})\right]}{\partial \mathrm{t}},
\end{aligned}
$$

where $\mathrm{t}$ and $\boldsymbol{f}$ denote the different time scales of observation with $(\mathrm{t}<<\boldsymbol{t})$. $\mathrm{dK}_{3} / \mathrm{d} \boldsymbol{I}$ follows changes in $\psi_{2}(\boldsymbol{l})$ and is given by the time dependence of the growing film $\mathrm{u}_{2}(\boldsymbol{l})$, whereas the second and third terms follow the time dependence of $\mathrm{d}_{1}(\mathrm{t})$ and $\varepsilon_{1}(\mathrm{t})$ both varying in time due to the periodic precursor exposure of the surface, causing cyclic changes in thickness and composition in the SRL, expressed in the boundary condition $\Phi_{1}(\mathrm{t})=\Phi_{1}\left(\mathrm{t}+\mathrm{T}_{\mathrm{CS}}\right)$. The cycle sequence time $\mathrm{T}_{\mathrm{CS}}$ is the order of a few seconds with precursor exposure times of tens of a second. Since the variations in time differ by more than a factor 100 , changes in $R_{3}$ for small time units $(t \leq 3 \mathrm{~s})$ can be set as linear.

For all simulations shown below the following parameters were kept constant, unless otherwise posted:

- complex dielectric function for the substrate of $\varepsilon_{\mathrm{Si}}=(15.25,0.17)[16]$,

- complex dielectric function for the bulk film of $\varepsilon_{\mathrm{GaP}}=(11.10,0.1)[17]$,

- wavelength $\lambda=6328 \AA$,

- cycle sequence $3 \mathrm{sec}$, with a TBP pulse from 0-0.8 sec, a TEG pulse from 1.4-1.8 sec and

- a growth rate $1 \AA ̊$ s. 
Figure 2 shows the simulated reflectivity for a 4-layer stack built up by the substrate Si with $\varepsilon_{3}(\lambda=632.8 \mathrm{~nm})=(15.25,0.17)$, the growing GaP film with $\varepsilon_{2}(\lambda=632.8 \mathrm{~nm})=(11.1,0.1)$ and an average growth rate of $1 \AA / \mathrm{sec}$, a periodic modulated SRL with $\varepsilon_{1}(\lambda=632.8 \mathrm{~nm})=(9.5,2.5)$ a minimal thickness of $d_{1 \min }=0.5 \AA\left(->\phi_{1 \min }\right)$ and maximal thickness of $d_{1 \max }=5 \AA\left(->\phi_{1 \max }\right)$, and an ambient with $\varepsilon_{0}=1$. Also drawn in are the calculated changes of $\left[\mathbf{R}_{4}(t)-R_{4}\left(\phi_{1 \min }\right)\right\rfloor$ and the four layer stack $\partial R_{4}(t) / \partial t$. The values for the average dielectric function of the SRL are chosen such that it matches typical experimental results for $\mathrm{GaP}$ on $\mathrm{Si}(001)$ monitored at an angle of incidence of 70deg. Under these specific growth conditions $\operatorname{Re}\left(\varepsilon_{1}\right)$ is smaller than $\operatorname{Re}\left(\varepsilon_{2}\right)$ with a significant absorption of $\operatorname{Im}\left(\varepsilon_{1}\right)=2.5$.

Figure 2:

Simulation of the temporal evolution of the PR signal in a four layer stack model. Also shown are the first derivative of the PR signal and SRL contributions to the reflectance (see text).

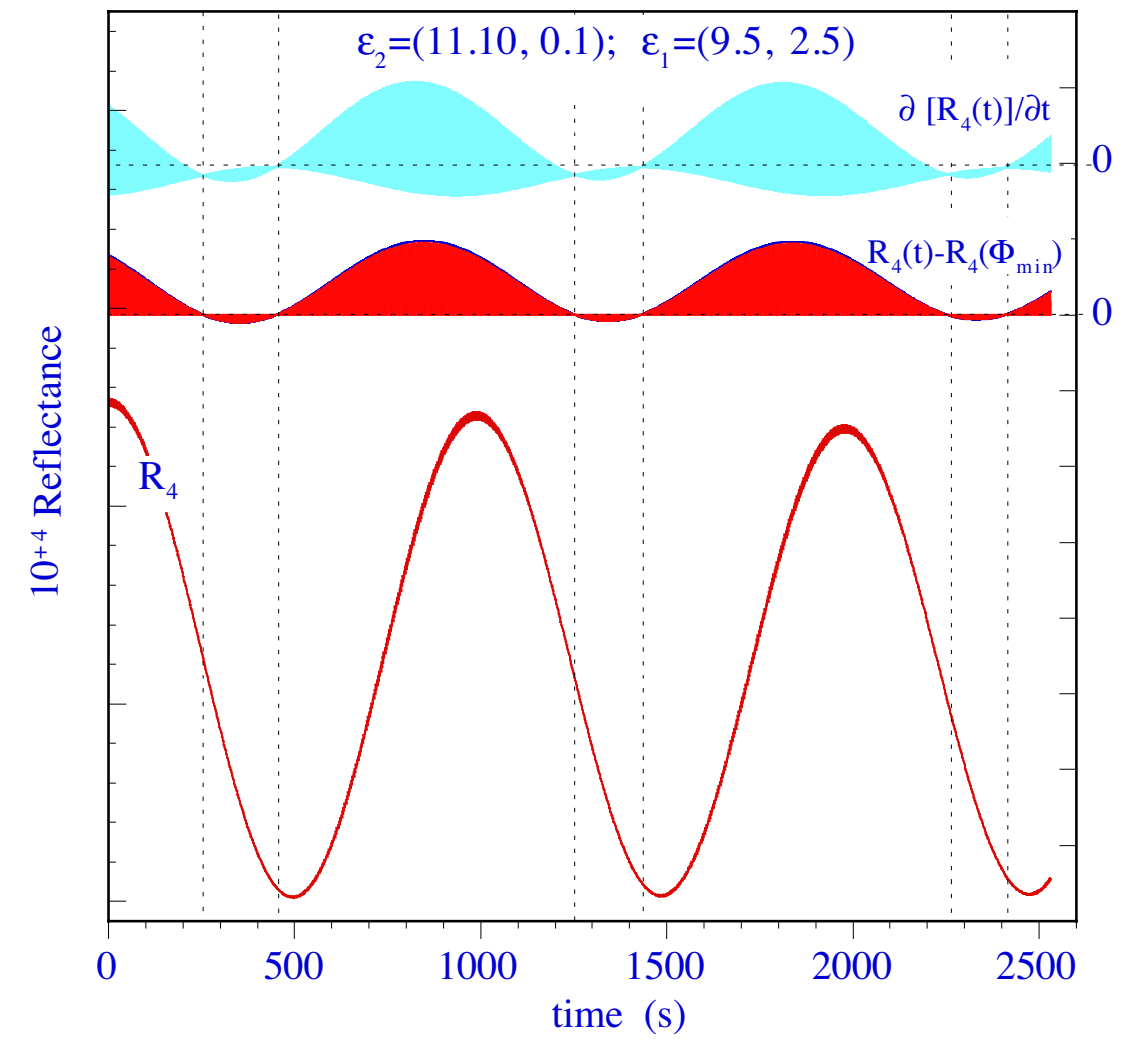

To evaluate the sensitivity and accuracy with which the average dielectric function $\varepsilon_{1}$ can the extracted, the complete parameter space of $\operatorname{Re}\left(\varepsilon_{1}\right)$ and $\operatorname{Im}\left(\varepsilon_{1}\right)$ as a function of the optical properties of the underlying film has to be evaluated. Figure 3 shows the shift of the turning points as a function of $\operatorname{Im}\left(\varepsilon_{1}\right)$ for two values of $\operatorname{Re}\left(\varepsilon_{1}\right)$. In fig.3(a) the turning points are located at the falling flank of the interference oscillation. With no absorption in the SRL, the position of the turning points coincide with the extreme of the interference oscillations. With increasing absorption $\operatorname{Im}\left(\varepsilon_{1}\right)>0$, the turning points closing together, shifted downwards along the falling flank of the interference oscillation until for $\operatorname{Im}\left(\varepsilon_{1}\right)>3$ only a local minimum remains. For $\operatorname{Re}\left(\varepsilon_{1}\right)$ values smaller 7 the same effect is observed in the raising flank of the interference oscillation as demonstrated for $\operatorname{Re}\left(\varepsilon_{1}\right)=6$ in fig. $3(\mathrm{~b})$. The influence of $\operatorname{Re}\left(\varepsilon_{1}\right)$.on the relative position of the turning points is shown in fig. 4(a) for a constant $\operatorname{Im}\left(\varepsilon_{1}\right)$. Here, the turning points are located at the increasing flank of the interference oscillation $\operatorname{Re}\left(\varepsilon_{1}\right)=6$ (lowest curve) and get shifted to the left towards the falling flank of the interference oscillation for $\operatorname{Re}\left(\varepsilon_{1}\right)=6$ (top curve). For intermediate values of $\operatorname{Re}\left(\varepsilon_{1}\right)$ in the range of 6.9 to 8.9 only a local minimum and no turning points are observed, which is due to the fact that $\mathrm{R}_{4}\left[\phi_{1-\max }\right]$ is always larger $\mathrm{R}_{4}\left[\phi_{1 \_} \_\mathrm{min}\right]$.

The influence of the surface reaction layer coverage, $d_{1}$ is shown in fig. $4(b)$, where the maximal coverage is varied from $2 \AA$ up to $10 \AA$. As it can be seen, the coverage has no influence on the position of the turning points relative to the interference oscillation. An increase/decrease in surface coverage only affects the amplitude of the observed fine structure or its derivative. This phenomena is due to the fact that the locations of the turning points are characterized by the values of the reflectance coefficients $r_{01}, r_{12}$ and $r_{23}$ which do not included the thickness $d_{1}$, whereas the 

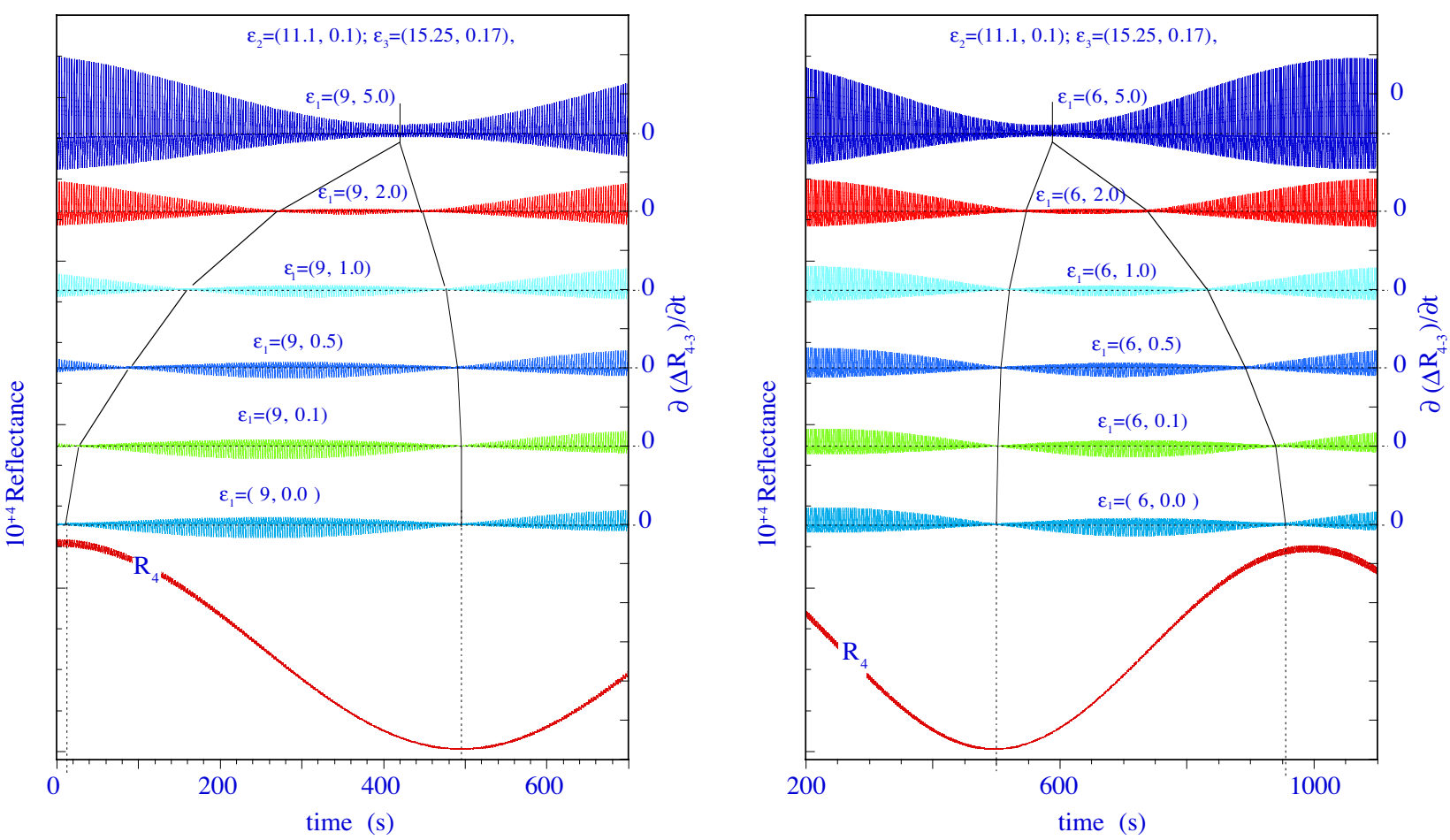

Figure 3. (a) Shift of the turning point positions along the falling flank of the interference oscillation with increasing absorption the SRL for $\operatorname{Re}\left(\varepsilon_{1}\right)=9$. and (b) Shift of the turning point positions along the raising flank of the interference oscillation with increasing absorption of the SRL for $\operatorname{Re}\left(\varepsilon_{1}\right)=6$.
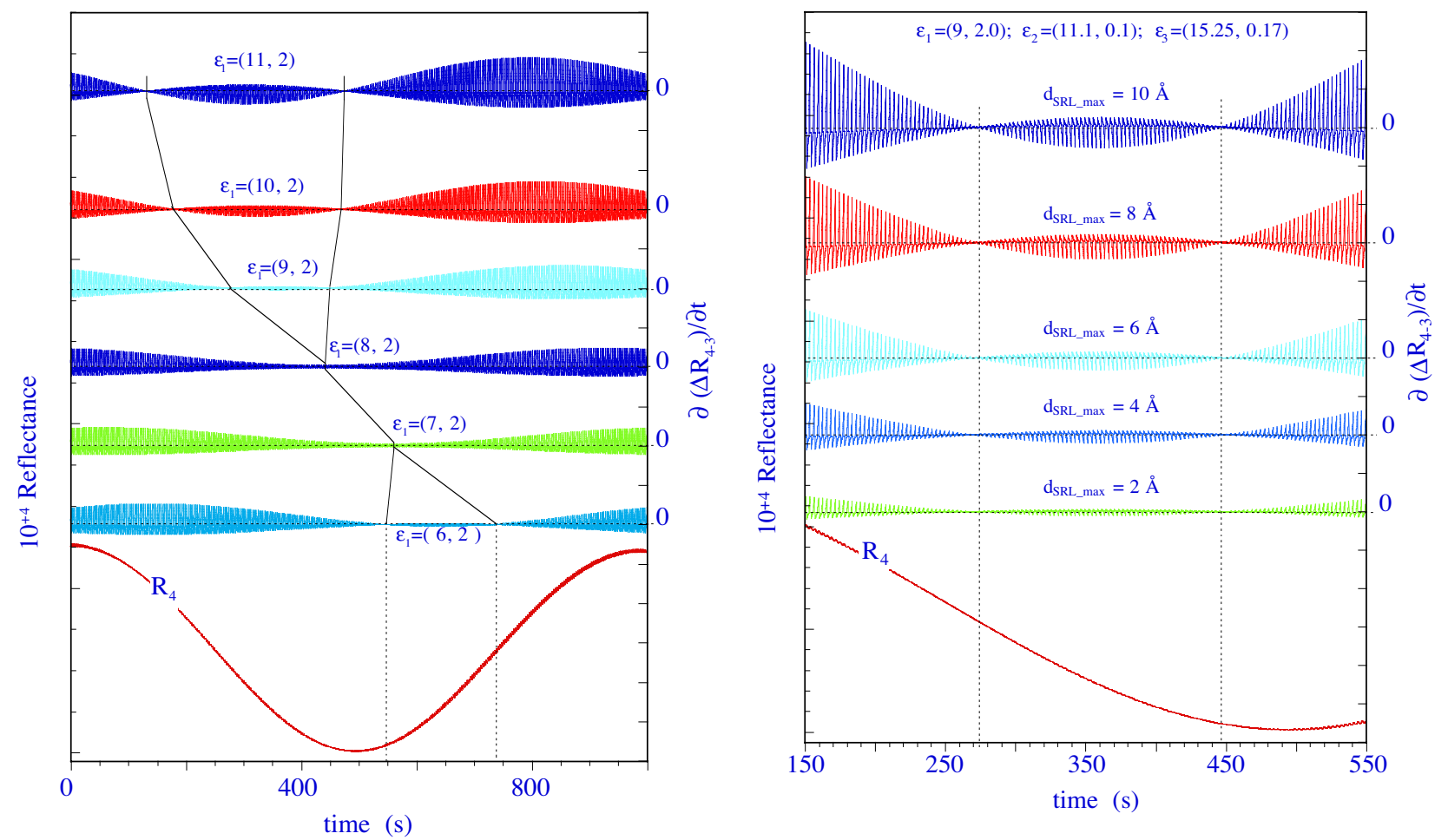

Figure 4. (a) Change of turning point positions relative to the interference extreme as a function of $\operatorname{Re}\left(\varepsilon_{1}\right)$ and (b): Influence of surface coverage at the point positions relative to the interference extreme and the amplitude of the signal 
amplitude of the signal is correlated to the phase factors $\phi_{1}(\mathrm{t})$ and $\phi_{2}(\mathrm{t})$. Utilizing these features allows extraction of the average dielectric function of the SRL, $\varepsilon_{1}$, from the locations of the turning point independent from the surface coverage. Once $\varepsilon_{1}$ is computed, the amplitude of the fine structure and/or its derivative can be used in the next step to calculate the average surface coverage for the SRL. In a more detailed model, the average dielectric function $\varepsilon_{1}$ has to be replaced by the time-dependence of $\varepsilon_{1}$, linking the polarizability of the SRL species to the decomposition processes of the SRL constituent as described by the activity coefficient for each constituent. Gaining access to these data will be a challenge for further work.

\section{CONCLUSION}

Real-time optical monitoring by p-polarized reflectance in combination with laser light scattering has been demonstrated to be a powerful tool to characterize growth processes as well as the surface chemistry under pulsed chemical beam growth conditions. We have shown that the average optical properties of an ultra-thin SRL can be quantitatively accessed under PCBE growth conditions by analyzing the amplitude modulation of fine structure, which exhibit turning points at which the response to the first precursor pulse changes sign. Comparison of four layer stack simulations with experimental results revealed an average dielectric function of $\varepsilon_{1}=(9.5,2.5)$ with an average thickness of $5 \AA$ for the SRL for GaP growth with $3 \mathrm{sec}$ pulse cycle time.

\section{Acknowledgments}

This work has been supported by the NSF Grant CDR 8721505, the DOD-MURI Grant F49620-95-1-0447 and the NASA Cooperative Agreement NCC8-95.

\section{References}

1. W. G. Breiland and K. P. Killeen, Mater. Res. Soc. Proc. 406, Boston, MA, USA, 29 Nov-2 Dec 1995, ed. by O. J. P. Glembocki, S.W.; Pollak, F.H.; Crean, G.M.; Larrabee, G., p.99-104 (1995).

2. W. G. Breiland and K. P. Killeen, J.Appl. Phys. 78 p.6726 (1995).

3. K. P. Killeen and W. G. Breiland, J. Electronic Materials, 23(2), 179-183 (1993).

4. S. D. Murthy, I. B. Bhat, B. Johs, S. Pittal and P. He, J. Elect. Mater. 24, 445-449 (1995).

5. D.E. Aspnes, J.P. Harbison, A.A. Studna and L.T. Florez, Appl. Phys. Lett. 52(12), 957-9 (1988).

6. N. Dietz, A. Miller, J. T. Kelliher, D. Venables and K. J. Bachmann, J. Crystal Growth 150 (14) 691-695 (1995).

7. N. Dietz, A. Miller and K. J. Bachmann, J. Vac. Sci. Technol. A 13, 153-155 (1995).

8. N. Dietz and K. J. Bachmann, MRS Bulletin 20, 49-55 (1995).

9. N. Dietz, U. Rossow, D. Aspnes and K.J. Bachmann, JEM 24(11) 1571-76 (1995).

10. K. J. Bachmann, N. Dietz, A. E. Miller, D. Venables and J. T. Kelliher, J. Vac. Sci. \& Technol. A 13, p.696-704 (1995).

11. N. Dietz and K. J. Bachmann, Vacuum 47, 133-40 (1996).

12. K. J. Bachmann, U. Rossow, N. Sukidi, H. Castleberry and N. Dietz, J. Vac. Sci. Technol. B 14, 3019 (1996)

13. U. Rossow, N. Dietz, K. J. Bachmann and D. E. Aspnes, J. Vac. Sci. Technol. B 14, 3040 (1996)

14. N. Dietz, N. Sukidi, C. Harris and K. J. Bachmann, JVST B, accepted (1997).

15. K. J. Bachmann, N. Sukidi, C. Hoepfner, C. Harris, S. LeSure, N. Dietz, H. T. Tran, S. Beeler, K. Ito and H. T. Banks, J. Crystal Growth, accepted (1997).

16. D. F. Edwards, in "Handbook of Optical Constants", ed. by E. D. Palik (Academic Press, 1985) 547 (1985).

17. A. Borghesi and G. Guizzetti, in "Handbook of Optical Constants", ed. by E. D. Palik (Academic Press, 1985) 445 (1985). 